International Journal Or Mechanical Engineering And Information Technology

An Official Publication of $18 \mathrm{M}$ Publication

\title{
Parametric Analysis of Hot Rolling Process
}

Authors

Prakhar Chansauria ${ }^{1}$, Shailendra Kumar ${ }^{2}$, Ashish Gupta ${ }^{3}$,

${ }^{1}$ Department of Mechanical Engineering, MANIT, Bhopal (MP), India

${ }^{2,3}$ Department of Mechanical Engineering, IIT Bombay, Powai, Mumbai, India

E-mail: prakharmahoba@gmail.com, shailendra113.iitb@gmail.com, ashishiitb99@gmail.com,

\begin{abstract}
In today's scenario finite element simulation has become an important tool in the manufacturing industry. Rolling process plays an important role in manufacturing of different parts with a long range variety of dimensions. In the process of rolling, the internal raw material transform into desired shape by passing through one or more pairs of rolls. The main aim of this paper is to investigate the influence of Modelling and Simulation of different parameters such as geometry of the slab, friction between work-rolls and slab, rotational speed of work-roll, percentage of thickness reduction of slab while using rolling process. Two dimensional rolling models are used routinely in most aluminium manufacturing companies. In present research, a two dimensional elasto-plastic Finite Element model for hot rolling of a plate of aluminium has been developed to study the behavior of the paticular material under different coefficients of friction, different roller diameters and different initial thickness of the metal plate for obtaining a particular final thickness of the rolled plate. The individual effects of coefficient of friction, roller radius, and initial thickness of plate on maximum stress, strain, strain rate, equivalent plastic strain and reaction force have been obtained. The results of finite element simulation can be used to investigate the effects of parameters on mechanical properties and product integrity.
\end{abstract}

Keywords: Hot Rolling, Von Misses Stress, Plastic Equivalent Strain, Reaction Force, FEM

\section{INTRODUCTION}

The rolling process is popular process in manufacturing industries. A two-dimensional elasto plastic Finite Element model using Abaqus 6.10 Software, for single pass hot rolling of a plate of Aluminium-2024 has been developed to study the behavior of the material under different coefficients of friction and different roller diameters for obtaining optimized parameters.

It is focused on study on parameter optimization of hot rolling process using FEM. For example, Chumachenko E. N. et al.(2010) developed the finite-element modeling of rolling in passes based on the $2 \mathrm{D}$ method. The approach was based on the assumption that strain rate in rolling direction is constant within each cross section of the deformation zone. The model was used to develop the computer system SPLEN (Rolling), which makes it possible to predict the deformation of metal during its rolling in passes. Esteban L. et al.(2007) studied the effect of different variables of the rolling operation on the lateral spread in the first pass of the finishing mill. A simplified 3D finite element model was used to calculate the stress and strain fields in the rolling gap using the constitutive equations for the material calculated from torsion tests performed at high temperatures. Farhat-Nia F. et al.(2006) simulated Asymmetric sheet rolling by using an elastic-plastic arbitrary Lagrangian-Eulerian (ALE) finite element method. The ALE finite element approach predicts the curvature development due to inequality in work roll/plate surface finish (interface friction) and speed mismatch assuming an isotropic work hardening material. Hambleton J.P. et al(2009) compared predictions of deformation and horizontal (drag) force resulting from three- and two-dimensional numerical simulation of a torque-free (towed) wheel operating on ductile material. The material was modeled as elastic/perfectly plastic with the von Mises yield condition, with focus on plastic rather 
than elastic effects. It was shown that twodimensional analysis of a rolling wheel cannot readily be applied to a narrow wheel to predict wheel penetration, although horizontal forces from three- and two- dimensional simulations follow a similar trend.

\section{GOVERNING EQUATIONS FOR THE STRESS PARAMETERS:}

The equation that governs the conditions of equilibrium of both the plastically deforming strip and the elastically loaded rolls is the following:

$$
\frac{\partial \sigma_{i j}}{\partial x_{j}}+\rho \cdot b_{j}=\rho \frac{\partial v_{j}}{\partial t}
$$

Where,

$\sigma_{\mathrm{ij}}=$ components of the Cauchy stress tensor

$b_{j}=$ components of body forces vector

$\mathrm{v}_{\mathrm{j}}=$ components of velocity vector

$\rho=$ density of material

In the rectangular Cartesian coordinate system, if the body force is neglected, the equilibrium equations are described by:

$$
\begin{aligned}
& \frac{\partial \sigma_{x}}{\partial x}+\frac{\partial \tau_{y x}}{\partial y}+\frac{\partial \tau_{z x}}{\partial z}=0 \\
& \frac{\partial \tau_{x y}}{\partial x}+\frac{\partial \sigma_{y}}{\partial y}+\frac{\partial \tau_{y z}}{\partial z}=0 \\
& \frac{\partial \tau_{x z}}{\partial x}+\frac{\partial \tau_{y z}}{\partial y}+\frac{\partial \sigma_{z}}{\partial z}=0
\end{aligned}
$$

\section{FRICTION MODEL PARAMETERS:}

A contact boundary condition describing the mechanical interaction of the rolled plate and the roller was imposed along the roller/plate interface. The friction at the interface for the contact area is proportional to the normal force as:

$$
\tau_{\text {crit }}=\mu \mathrm{P}
$$

where $\tau_{\text {crit }}$ (in Pa) is critical shear stress, $\mu$ is the coefficient of friction and $\mathrm{P}$ is contact pressure. In the basic Coulomb's friction model, two contact surfaces can carry shear stresses up to a certain magnitude $\left(\tau_{\text {crit }}\right)$ across their interface before they start sliding relative to each other. The state is known as sticking. Once the equivalent shear stress exceeds $\left(\tau_{\text {crit }}\right)$, the contact and target surfaces will slide relative to each other. This state is known as sliding.

Deformation symmetry in the plate is maintained through a zero displacement boundary condition in the thickness in the direction along the plate centerline. This allows a reduction in geometric complexity and reduces computational time.

\section{MATERIAL SELECTION \& CHEMICAL COMPOSITION}

ALLOY 2024: Alloy 2024 was introduced by Alcoa in 1931 as an alclad sheet. It was the first $\mathrm{Al}-\mathrm{Cu}-\mathrm{Mg}$ alloy to have a yield strength approaching $50,000-\mathrm{Kg} / \mathrm{cm}^{2}$ and generally replaced 2017-T4 (Duralumin) as the predominant 2XXX series aircraft alloy. With its relatively good fatigue resistance, especially in thick plate forms, alloy 2024 continues to be specified for many aerospace structural applications. It is considered as the "aircraft" alloy because of its strength. Good machinability but only fair corrosion resistance. Not recommended for brazing or soldering. Alloy 2024 is available in bar and alclad sheet and plate product forms in the annealed state and several tempers is described by Alcoa Mill Products.

Chemical Composition of aluminium 2024 given below

Si $0.50 \%$

$\mathrm{Zn} 0.25 \%$

Fe $0.50 \%$

Ti $0.15 \%$

$\mathrm{Cu} 3.8-4.9 \%$

Mn $0.30-0.9 \%$

$\mathrm{Mg} 1.2-1.8 \%$

Cr $0.10 \%$

Aluminum 93.2\%[36] 


\section{PARAMETRIC CALCULATION USING ABAQUS}

\section{1 a) VON Mises Stress:}

The effect of variation in Initial Height of the Aluminium plate on von Mises Equivalent Stress at $50 \mathrm{~mm}$ roller radius and different values of percentage reduction is shown in the figures below:

(a) At Roller Diameter $50 \mathrm{~mm} \&$ reduction is $5 \%$ :

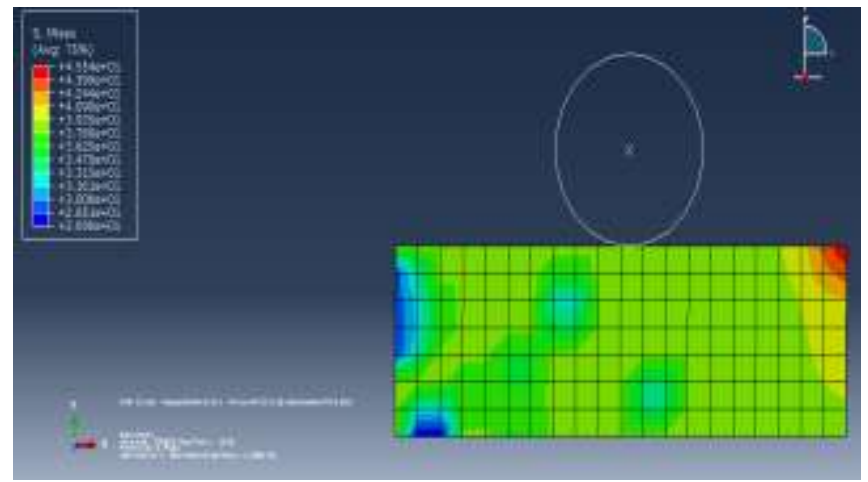

Fig.1 Effect of von-Mises Stress at Roller Diameter $50 \mathrm{~mm} \&$ reduction is $5 \%$

(b) At Roller Radius $50 \mathrm{~mm} \&$ reduction is $10 \%$ :

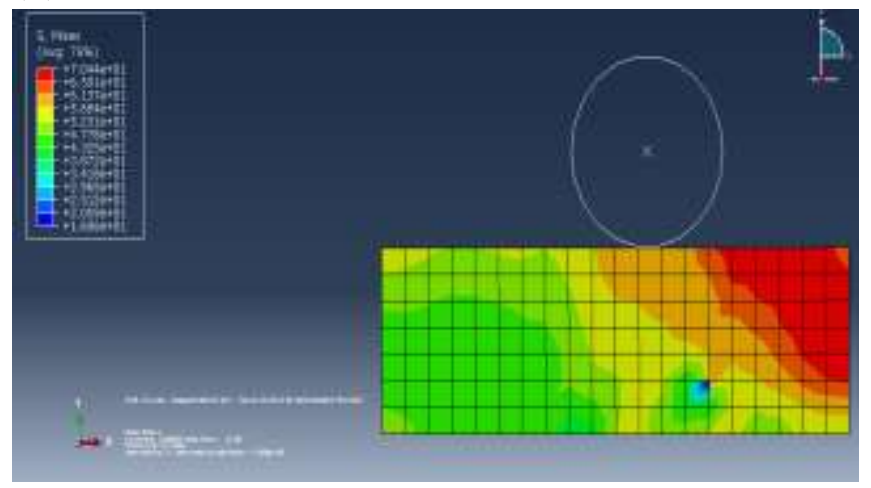

Fig. 2 Effect of von-Mises Stress at Roller Radius $50 \mathrm{~mm} \&$ reduction is $10 \%$

(c) At Roller Diameter $50 \mathrm{~mm} \&$ reduction is $15 \%$ :

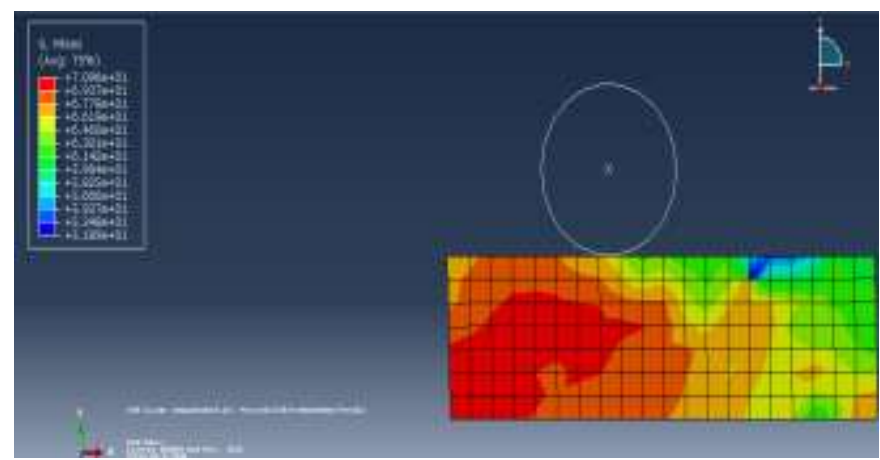

Fig.3 Effect of von-Mises Stress at Roller Diameter $50 \mathrm{~mm} \&$ reduction is $15 \%$
Thus, we see that the effect of increase in coefficient of friction has a small effect on the von Mises Equivalent Stress at a particular initial height of the Aluminium plate. When the reduction is increased from $5 \%$ to $15 \%$, the von Mises Stress increases linearly at a particular initial height of the plate, but the increase is much smaller as compared to the when the initial height of the Aluminium plate is increased. Thus, the effect of increase in initial height of the plate is more pronounced than increase in coefficient of friction.

\section{1 b) Equivalent Plastic Strain:}

The effect of variation in Initial Height of the Aluminium plate on Equivalent Plastic Strain at same roller radius and different values of reduction percentage is shown in the figures below:

(a) At Roller Radius $50 \mathrm{~mm} \&$ reduction is $5 \%$ :

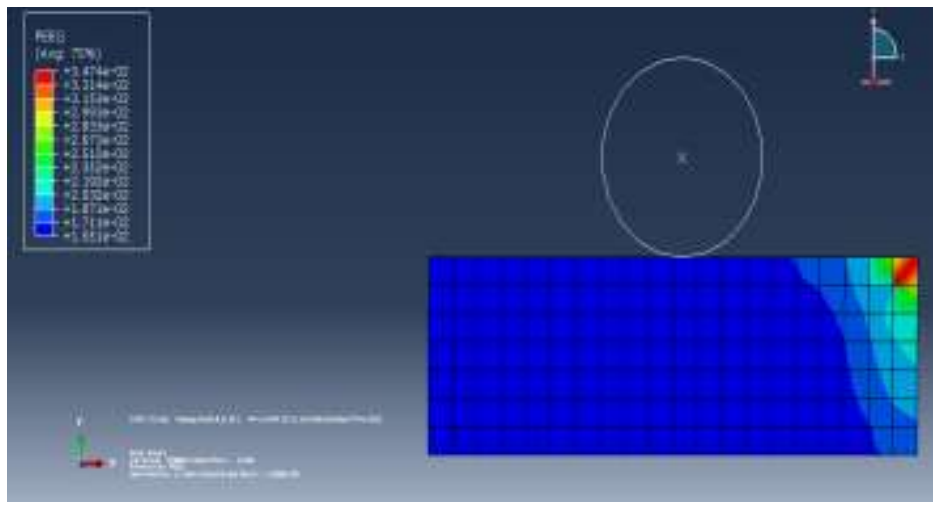

Fig. 4 Effect of Equivalent Plastic Strain at Roller Radius $50 \mathrm{~mm} \&$ reduction is $5 \%$ :

(b) At Roller Radius $50 \mathrm{~mm} \&$ reduction is $10 \%$ :

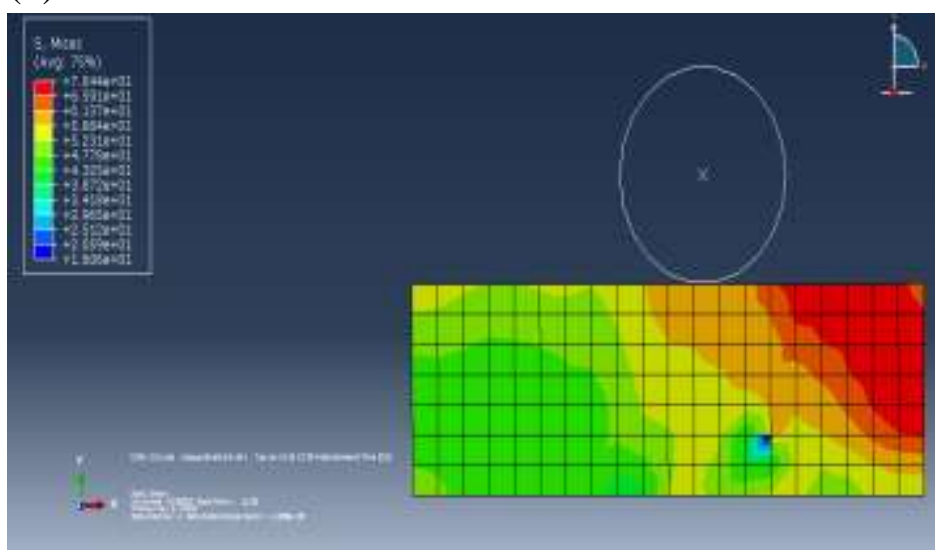

Fig. 5 Effect of Equivalent Plastic Strain at Roller Radius $50 \mathrm{~mm} \&$ reduction is $10 \%$ 
(c) At Roller Diameter $50 \mathrm{~mm} \&$ reduction is $15 \%$ :

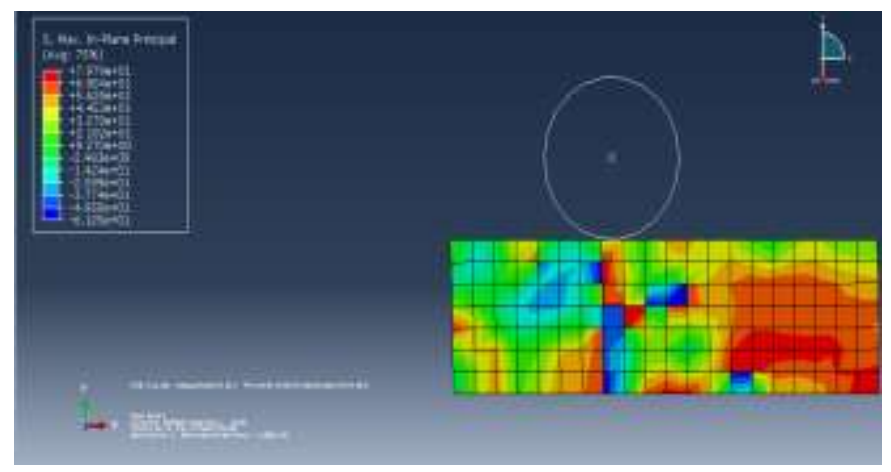

Fig. 6 Effect of Equivalent Plastic Strain at Roller Radius $50 \mathrm{~mm} \&$ reduction is $15 \%$

In the case of Equivalent Plastic Strain as well, the increase in reduction from $5 \%$ to $15 \%$, in a small linear increase in the magnitude of Equivalent Plastic Strain at a particular initial height of the plate. However, the increase is much smaller as compared to the increase in Equivalent Plastic Strain with increase in the initial height of the plate. Therefore, the effect of increase in initial height of the Aluminium plate is more pronounced than the effect of increase in coefficient of friction.

\section{1 c) Reaction Force:}

The effect of variation in Initial Height of the Aluminium plate on the reaction force at same roller radius and different values of percentage reduction is shown in the figures below:

(a) At Roller Radius $50 \mathrm{~mm} \&$ reduction is $5 \%$ :

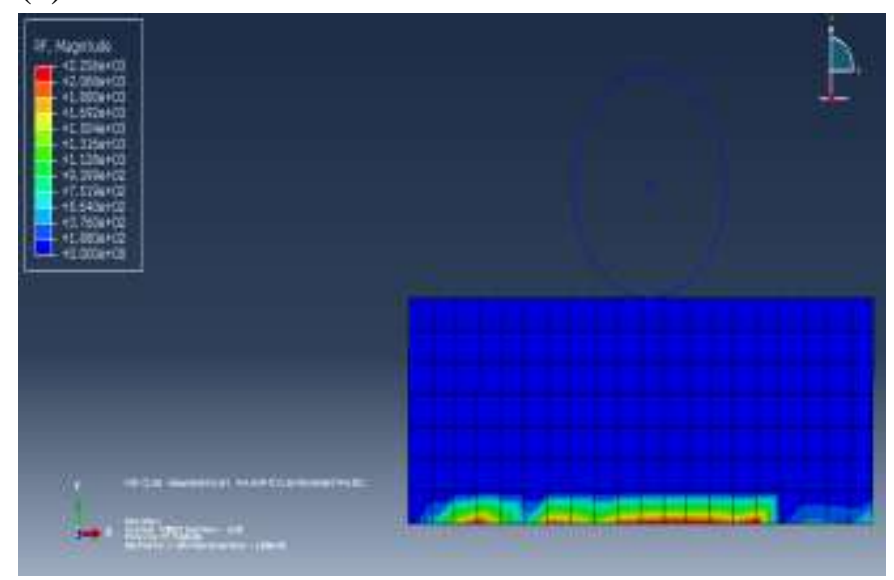

Fig. 7 Effect of Reaction Force at Roller Radius $50 \mathrm{~mm} \&$ reduction is $5 \%$ (b) At Roller Radius $50 \mathrm{~mm} \&$ reduction is $10 \%$ :

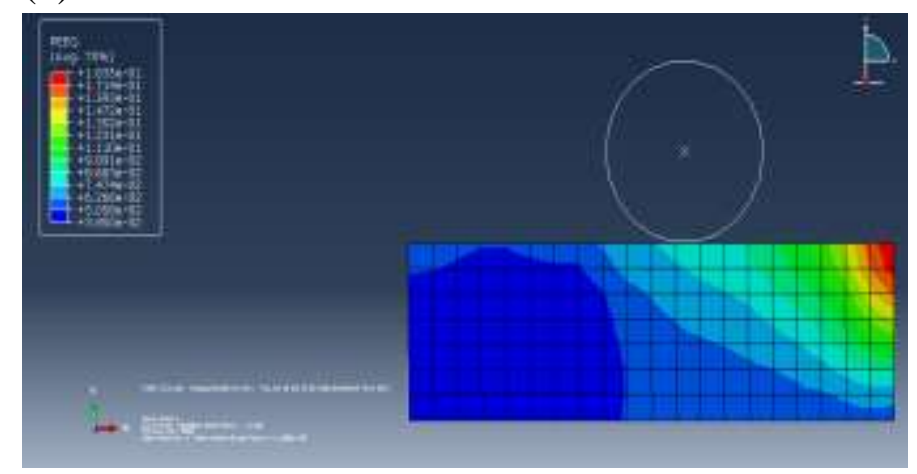

Fig. 8 Effect of Reaction Force at Roller Radius $50 \mathrm{~mm} \&$ reduction is $10 \%$

(c)At Roller Diameter $50 \mathrm{~mm} \&$ reduction is $15 \%$ :

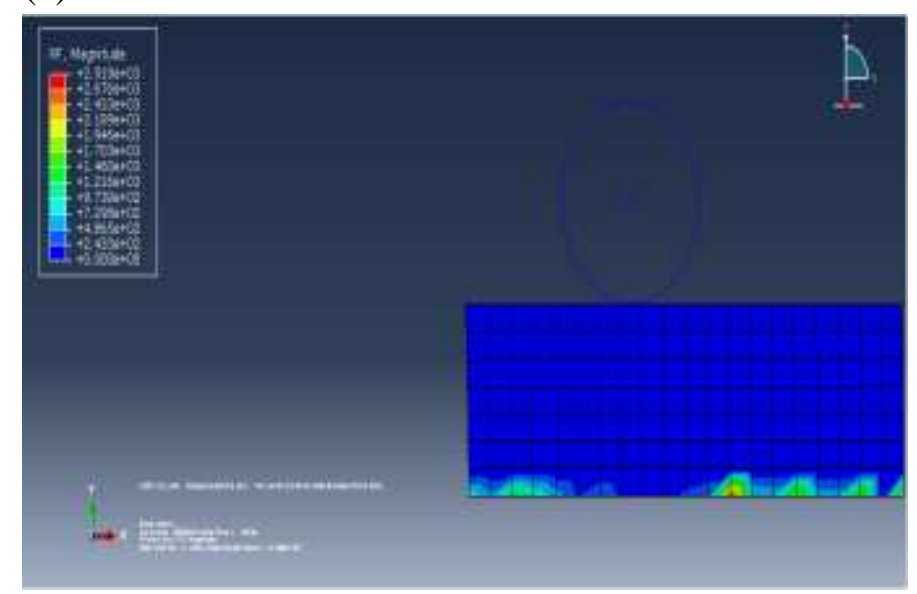

Fig. 9 Effect of Reaction Force at Roller Radius $50 \mathrm{~mm} \&$ reduction is $15 \%$

In the case of Reaction Force as well, the effect of increase in percentage reduction is smaller as compared to the effect of increase in initial height of the Aluminium plate. Also, the increase in Reaction Force with increase in coefficient of friction is more pronounced at higher reduction. However the increase in Reaction Force with increase in initial height of the plate is not as much as it is in the case of von Mises Equivalent Stress and Equivalent Plastic Strain.

\section{CONCLUSIONS:}

It was found that the effect of increase in coefficient of friction has a small effect on the Von Mises Equivalent Stress at a particular initial height of the Aluminium plate.

The effect of increase in coefficient of friction is smaller than the effect of increase in Roller Radius on the von Mises Equivalent Stress. However, the 
increase in von Mises Stress with increase in Roller Radius is not as much as it is with increase in Initial Height of the plate.

\section{REFERENCES}

1. ChumachenkoE. N" Mathematical modeling and energy conservation for rolling in passes" Metallurgist, Vol. 54, Nos. 7-8, 2010

2. Esteban L., Elizalde M.R., OcanaI."Mechanical characterization and finite element modeling of lateral spread in rolling of low carbon steel" Journal of
Materials Processing Technology 183(2007) 390-398

3. Farhat-Nia F., Salimi M., Movahhedy M.R. "Elasto - plastic finite element simulation of asymmetrical plate rolling using an ALE approach" Journal of Materials Processing Technology 177 (2006) 525-529

4. Hambleton J.P., Drescher A. "On modeling a rolling wheel in the presence of plastic deformation as a three or twodimensional process" International Journal of Mechanical Sciences 51 (2009) 846855 